\title{
MiR-34a, miR-21 and miR-23a as potential biomarkers for coronary artery disease: a pilot microarray study and confirmation in a $\mathbf{3 2}$ patient cohort
}

Hui Han ${ }^{1,3}$, Guangjin $\mathrm{Qu}^{1,3}$, Chenghua Han ${ }^{2}$, Yuhong Wang ${ }^{1}$, Tingting Sun ${ }^{1}$, Fengqing $\mathrm{Li}^{1}$, Junxiao Wang ${ }^{1}$ and Shanshun Luo ${ }^{1}$

The aim of this study was to investigate the expression of circulating microRNAs (miRNAs) in apolipoprotein E (apoE) knockout mice $\left(a p o E^{-l-}\right.$ ) and to validate the role of these miRNAs in human coronary artery disease (CAD). Pooled plasma from 10 apoE $\mathrm{E}^{-/-}$mice and 10 healthy C57BL/6 (B6) mice was used to perform the microarray analysis. The results showed that miR-34a, miR-21, miR-23a, miR-30a and miR-106b were differentially expressed in apoE $\mathrm{E}^{-/-}$mice, and these expression changes were confirmed by real-time quantitative reverse-transcription PCR. Then, miR-34a, miR-21, miR-23a, miR-30a and miR-106b were detected in the plasma of 32 patients with CAD and of 20 healthy controls. Only miR-34a, miR-21 and miR-23a were significantly differentially expressed in the plasma of CAD patients (all $P<0.01$ ). In conclusion, miR-34a, miR-21 and miR-23a were elevated in CAD patients, which means that these miRNAs might serve as biomarkers of CAD development and progression.

Experimental \& Molecular Medicine (2015) 47, e138; doi:10.1038/emm.2014.81; published online 6 February 2015

\section{INTRODUCTION}

Coronary artery disease $(\mathrm{CAD})$ is currently one of the major causes of death and illness, and its development is influenced by a number of environmental and genetic factors. ${ }^{1,2}$ Atherosclerosis, the main cause of $\mathrm{CAD}$, is caused by lipid-induced inflammation of the vessel wall that is orchestrated by a complex interaction between various cell types, such as endothelial cells and vascular smooth muscle cells. Lowering low-density lipoprotein cholesterol has revolutionized the treatment of CAD and has reduced the risk of cardiovascular events by up to $50 \% .^{2}$ Apolipoprotein E knockout mice $\left(\mathrm{apoE}^{-/-}\right)$are a well-established model that predictably develop nearly the entire spectrum of atherosclerotic plaques that are observed during atherogenesis in humans, in a similar manner as in humans. ${ }^{3-5}$

MicroRNAs (miRNAs) are a new class of highly conserved, non-coding small RNAs that usually consist of 19-24 nucleotides. These RNAs regulate gene expression on the posttranscriptional level by inhibiting the translation of protein from mRNA or by promoting the degradation of mRNA. The human genome may encode over 1000 miRNAs, ${ }^{6-8}$ and these endogenous gene expression inhibitors have been primarily described to have crucial roles in physiological and pathological processes, such as cell proliferation, differentiation, apoptosis and carcinogenesis. Various miRNAs have been shown to control vascular smooth muscle cell, endothelial cell and macrophage functions, and therefore to have a role in the progression of atherosclerosis. ${ }^{9-12}$ In addition, miRNAs modulate several pathways involved in plaque development, such as cholesterol metabolism and inflammation. ${ }^{13}$

Recent studies demonstrated that miRNAs can be detected in the blood and may be used as potential biomarkers of various diseases. $^{2}$ For example, miR-10a regulates pro-inflammatory phenotypes in atherosclerosis-susceptible endothelium both in vivo and in vitro. ${ }^{14}$ However, the exact involvement of miRNAs in atherosclerosis remains to be clarified.

In the present study, we identified the differentially regulated miRNAs in the plasma of $\mathrm{apoE}^{-/-}$and normal mice. Then, we

\footnotetext{
${ }^{1}$ Department of Gerontology, the First Hospital of Harbin Medical University, Harbin, China and ${ }^{2}$ Department of Natural Product Chemistry, the Daqing Campus of Harbin Medical University, Daqing, China

${ }^{3}$ They are co-first authors.

Correspondence: Dr S Luo, Department of Gerontology, the First Hospital of Harbin Medical University, Harbin, Heilongjiang Province 150001, China. E-mail: luoshanshunmed@163.com
}

Received 24 June 2014; revised 10 September 2014; accepted 18 September 2014 
measured the levels of these miRNAs in the plasma of CAD patients. Our results suggest that several miRNAs exhibit significantly altered expression profiles in the animal model as well as in CAD patients; therefore, these miRNAs might serve as biomarkers for the disease.

\section{MATERIALS AND METHODS}

\section{Animal and human subjects}

Ten apoE $\mathrm{E}^{-1-}$ mice and 10 healthy male C57BL/6 (B6) mice (Experimental Animal Center of the Peking University Health Science Center, Beijing, China) were fed until 8 months of age, using a standard food containing $6.5 \%$ fat, $19.5 \%$ protein and no cholesterol (Keao Xieli Feed, Beijing, China). This method was previously used with success to obtain atherosclerosis models in our laboratory. ${ }^{15}$

We recruited $32 \mathrm{CAD}$ patients (50-68 years old), who were confirmed by coronary angiography to have a $>70 \%$ coronary stenosis, at the First Hospital of Harbin Medical University (Harbin, China) between June 2012 and December 2012. We also recruited 20 healthy volunteers (47-71 years old) to serve as controls. These healthy control subjects had a normal electrocardiogram and no history of arterial hypertension, angina, $\mathrm{CAD}$ or peripheral artery disease.

The study was approved by the Ethical Committee of the Harbin Medical University. All volunteers provided written informed consent, and the procedure was conducted in adherence with all applicable state and university guidelines.

\section{Blood sampling}

Mice were anesthetized with sodium pentobarbital $\left(75 \mathrm{mg} \mathrm{kg}^{-1}\right.$, intraperitoneally). A tube was inserted through the sinus membrane of the eyeball using a gentle rotating motion and then rotated until the blood flowed; the maximum amount of blood was collected while carefully avoiding contamination. EDTA was added to the blood to avoid coagulation, and samples were stored at room temperature for no longer than $2 \mathrm{~h}$ before being centrifuged at $1200 \mathrm{~g}$ for $10 \mathrm{~min}$ at $4{ }^{\circ} \mathrm{C}$. The supernatant was transferred to $1.5-\mathrm{ml}$ Eppendorf tubes and centrifuged at $12000 \mathrm{~g}$ for $10 \mathrm{~min}$ at $4{ }^{\circ} \mathrm{C}$ to remove cellular components. The purified plasma was mixed with Trizol LS reagent (Invitrogen, Carlsbad, CA, USA) and then stored at $-80^{\circ} \mathrm{C}$. Plasma from 10 mice of each group was pooled for analysis.

For the human subjects, venous blood samples were obtained via antecubital venipuncture in the sitting position. Whole blood $(5 \mathrm{ml})$ was placed in EDTA-containing tubes and then centrifuged (1200 g for $10 \mathrm{~min}$ at $\left.4{ }^{\circ} \mathrm{C}\right)$. The supernatant was collected and centrifuged (12 $000 \mathrm{~g}$ for $10 \mathrm{~min}$ at $4^{\circ} \mathrm{C}$ ). Plasma was then obtained, and $1 \mathrm{ml}$ was rapidly subjected to RNA extraction.

\section{Isolation of total RNA}

Total RNA was extracted from plasma using a TRIzol LS RNA isolation kit (Invitrogen) according to the manufacturer's protocol. Total RNA quality was tested using the NanoDrop 1000 system (ThermoFisher Scientific, Wilmington, DE, USA), and the RNA was immediately stored at $-80^{\circ} \mathrm{C}$.

\section{Microarray analysis of miRNA}

Plasma RNA $(1 \mu \mathrm{g})$ from apoE ${ }^{-1-}$ and control mice were separately labeled using the miRCURY Hy3/Hy5 Power Labeling Kit (Exiqon, Vedbaek, Denmark) and hybridized on the miRCURY LNA Array v.16.0 (Exiqon). After washing, the slides were scanned using the Axon GenePix 4000B microarray scanner (Axon Instruments, Foster City, CA, USA), and the images were imported into the GenePix Pro 6.0 software (Axon) for grid alignment and data extraction. Replicated miRNAs were averaged, and miRNAs with intensities $>50$ in all samples were used to calculate a normalization factor. Expression data were normalized using the median normalization value, and after normalization, a significant threshold value of a fold change $>3.0$ was used to define the upregulation or downregulation of miRNAs.

\section{Quantitative reverse-transcription PCR}

The expression levels of five miRNAs (miR-34a, miR-21, miR-23a, miR-30a and miR-106b) in apoE ${ }^{-1-}$ and B6 mice, as well as in 32 patients with $\mathrm{CAD}$ and 20 healthy control subjects were quantified using TaqMan MicroRNA assays (Applied Biosystems, Warrington, UK). Monitoring of miRNA-derived PCR products was performed on an ABI7500 quantitative PCR system (Applied Biosystems) and normalized to U6 RNA.

\section{Gene set enrichment analysis of miRNA targets in different pathways}

The miRNA (miR-34a, miR-21, miR-23a, miR-30a and miR-106b)target interactions were predicted using miRecords, which included 11 established miRNA target prediction programs: DIANA-microT, MicroInspector, miRanda, MirTarget2, miTarget, NBmiRTar, PicTar, PITA, RNA22, RNAhybrid and TargetScan. ${ }^{16}$ To improve the reliability of the miRNA target identification, only target genes predicted by at least four of the programs were selected for extraction. The predicted targets were then compared with a previously established microarray profile of differentially expressed mRNAs in apoE ${ }^{-1-}$ mice. ${ }^{15}$ Only genes identified in both analyses and whose expression was negatively correlated with the expression levels of the miRNAs related to CAD were considered as potential targets of the miRNAs. Functional enrichment analysis was performed using previously developed hypergeometric testing methods and Kyoto Encyclopedia of Genes and Genomes, which was designed to investigate the potential regulation of various pathways by differentially expressed miRNAs. ${ }^{17} \mathrm{Next}$, the extent of each gene's appearance in the refined gene sets of the Kyoto Encyclopedia of Genes and Genomes pathways was calculated using the method previously described in the DAVID Bioinformatics Database. ${ }^{14}$

\section{Statistical analyses}

All data are expressed as the means \pm s.d. ANOVA or Student's $t$-tests were used for statistical analyses. All miRNAs were considered to have significant differential expression if they were up- or downregulated by at least threefold. Statistical significance was determined when $P$-values were $<0.05$.

\section{RESULTS}

The levels of several miRNAs are significantly altered in the plasma of $\mathrm{apoE}^{-1-}$ mice

The average values for each miRNA after normalization were used for statistical analysis. We previously reported that 16 miRNAs were differentially expressed in apoE $\mathrm{E}^{-/-}$mice aorta samples compared with controls, and that 6 of these were upregulated and 10 were downregulated. ${ }^{15}$ In plasma, 14 miRNAs were differentially expressed in the $\mathrm{apoE}^{-1-}$ mice compared with the controls, and among these, six miRNAs were upregulated and eight were downregulated (Table 1). The miRNAs mmu-miR-34a, mmu-miR-21, mmu-miR-23a, mmu-miR-30a and mmu-miR-106b were differentially 
Table 1 Circulating microRNAs with altered expression levels in apoE $\mathrm{E}^{-/-}$compared with wild-type mice (Threshold value $>3$, microarray results)

\begin{tabular}{llc}
\hline Accession number & Name & Fold change \\
\hline MI0000584 & mmu-miR-34a & 3.46 \\
MIMAT0004531 & mmu-miR-135a-1* & 3.23 \\
MIMAT0004638 & mmu-miR-323-5p & 3.23 \\
MIMAT0003725 & mmu-miR-675-5p & 3.11 \\
MI0000247 & mmu-miR-204 & 3.06 \\
MI0000569 & mmu-miR-21 & 3.02 \\
MIMAT0017070 & mmu-miR-7a-2* & -4.21 \\
MIMAT0004898 & mmu-miR-654-3p & -3.63 \\
MI0000571 & mmu-miR-23a & -3.63 \\
MI0000685 & mmu-miR-10a & -3.40 \\
MI0000398 & mmu-miR-298 & -3.11 \\
MI0000140 & mmu-miR-15b & -3.07 \\
MI0000144 & mmu-miR-30a & -3.03 \\
MI0000407 & mmu-miR-106b & -3.02 \\
\hline
\end{tabular}

The miRNAs were differentially expressed in aorta samples, too.

expressed in plasma, as well as in previously published aorta samples. ${ }^{15}$

\section{Confirmation of miRNA expression by qRT-PCR}

To validate our microarray results, quantitative RT-PCR analysis of the levels of mmu-miR-34a, mmu-miR-21, mmumiR-23a, mmu-miR-30a and mmu-miR-106b in plasma was performed. The RT-PCR results showed that the expression of mmu-miR-34a and mmu-miR-21 was upregulated and that of mmu-miR-23a, mmu-miR-30a and mmu-miR-106b was downregulated (Figure 1), which was consistent with the microarray results.

miRNA expression in the plasma of CAD patients and healthy controls

We then determined the levels of these five miRNAs in the plasma of CAD patients and healthy controls. Table 2 shows the patients' characteristics. Of all physical and physiological features tested, only total and low-density lipoprotein cholesterol were higher in $\mathrm{CAD}$ patients compared with controls $(P=0.04$ and $P=0.04$, respectively); all other parameters were comparable between the two groups. The expression levels of the miRNAs were then assessed in CAD and healthy controls. As shown in Figure 2a, the levels of miR-34a and miR-21 were significantly higher in the plasma of CAD patients compared with controls, whereas that of miR-23a was lower (all $P<0.01$ ). There was no difference in the expression of miR-30a and miR-106b in CAD patients and healthy controls (both $P>0.05$; Figure $2 \mathrm{~b}$ ).

\section{DISCUSSION}

Aberrant expression of miRNAs has been identified in various diseases. Recent studies have demonstrated that miRNAs can be detected in the blood and that they may serve as potential biomarkers for a number of diseases. ${ }^{18,19}$ Moreover, the

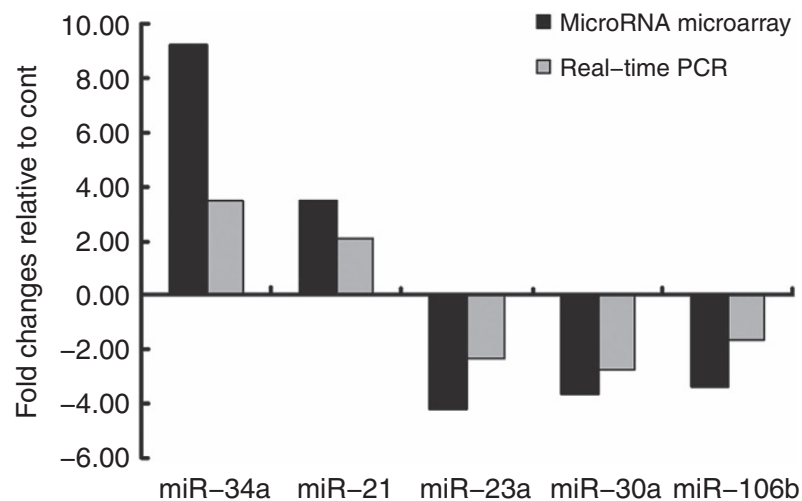

Figure 1 Relative expression levels of five selected microRNAs (miRNAs) in the plasma of $a p o E^{-/-}$mice compared with wild-type mice. The expression levels of the five miRNAs in apoE $E^{-/-}$mice were detected using TaqMan quantitative RT-PCR, and the relative fold changes compared with those of wild-type controls were compared with those from the microarray results.

Table 2 Clinical characteristics of patients with CAD and healthy controls

\begin{tabular}{|c|c|c|c|}
\hline & $C A D$ patients & Controls & P-value \\
\hline N & 32 & 20 & - \\
\hline Men & 32 & 20 & - \\
\hline Smoking status (yes/no) & $9 / 32$ & $5 / 20$ & 0.89 \\
\hline Age (years) & $67 \pm 11$ & $62 \pm 8$ & 0.14 \\
\hline BMI $\left(\mathrm{kg} \mathrm{m}^{-2}\right)$ & $24.1 \pm 3.7$ & $23.6 \pm 4.0$ & 0.33 \\
\hline Waist circumference $(\mathrm{cm})$ & $86.2 \pm 11.1$ & $84.2 \pm 13.7$ & 0.57 \\
\hline Systolic blood pressure (mm Hg) & $130.7 \pm 22.4$ & $135.0 \pm 26.7$ & 0.21 \\
\hline $\begin{array}{l}\text { Diastolic blood pressure } \\
(\mathrm{mm} \mathrm{Hg})\end{array}$ & $80.3 \pm 8.9$ & $78.4 \pm 10.2$ & 0.46 \\
\hline Total cholesterol (mmol $\left.{ }^{-1}\right)$ & $6.33 \pm 1.02$ & $4.81 \pm 1.76$ & $0.043^{*}$ \\
\hline HDL cholesterol (mmol I-1) & $1.12 \pm 1.16$ & $1.26 \pm 0.85$ & 0.08 \\
\hline LDL cholesterol (mmol l-1 $^{-1}$ & $5.12 \pm 0.36$ & $3.25 \pm 0.44$ & $0.037^{*}$ \\
\hline Glucose $\left(\mathrm{mmol} \mathrm{I}^{-1}\right)$ & $5.89 \pm 3.78$ & $2.67 \pm 2.26$ & 0.32 \\
\hline C-reactive protein $\left(\mathrm{mg}^{-1}\right)$ & $7.1 \pm 3.8$ & $5.4 \pm 3.6$ & 0.07 \\
\hline
\end{tabular}

Abbreviations: BMI, body mass index; CAD, coronary artery disease;

HDL, high-density lipoprotein; LDL, low-density lipoprotein.

${ }^{*}$ Difference is considered significant at $P<0.05$.

detection of circulating miRNAs can provide important novel information concerning diseases. In this study, a miRNA profile of the plasma of $\mathrm{apoE}^{-1-}$ mice was obtained, and we identified five miRNAs (miR-34a, miR-21, miR-23a, miR-30a and miR-106b) that were differentially expressed; this result was confirmed by quantitative reverse-transcription PCR. Furthermore, we examined the expression levels of these five circulating miRNAs in CAD patients compared with healthy controls, and we found that miR-34a, miR-21 and miR-23a may be biomarkers of CAD.

Hyperlipoproteinemia is recognized as one of the most important risk factors for the development of atherosclerosis, and the $\mathrm{apoE}^{-/-}$mouse model, even when given a normal diet, is a well-established model of hypercholesterolemia. ${ }^{20}$ This 
a

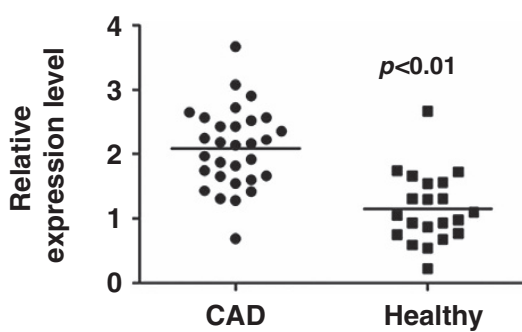

b

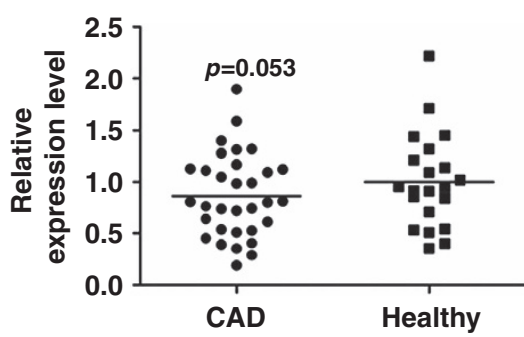

miR-21

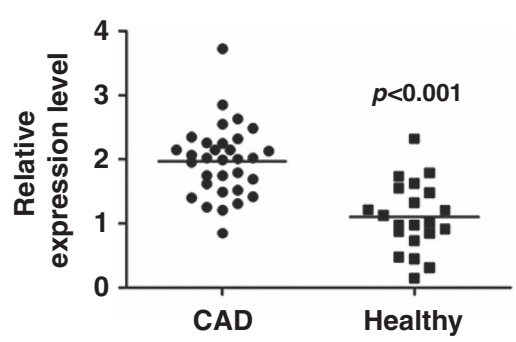

miR-106b

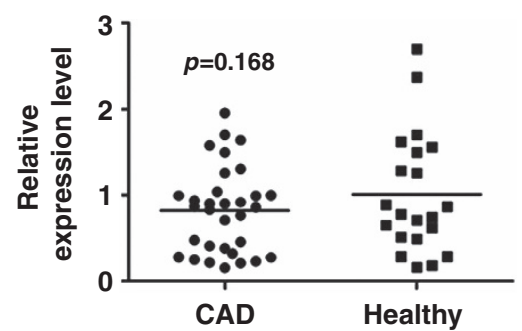

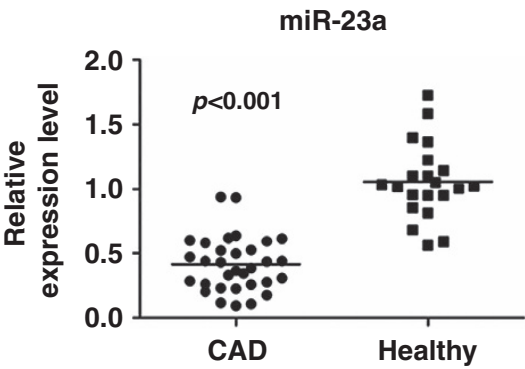

Figure 2 Circulating miRNAs in CAD patients and healthy controls. The expression of candidate miRNAs in the plasma of CAD patients compared with healthy controls, as determined by qRT-PCR. The expression levels of miRNAs were normalized to U6 expression. Horizontal lines indicate medians. P-values were calculated using two-sided Student $t$-tests. (a): miRNAs that are differentially expressed in CAD plasma $(P<0.01)$. (b): miRNAs whose expression is not different between CAD patients and controls $(P>0.05)$.

Table 3 Pathway enriched in predicted targets of miR-34a, miR-21, miR-23a, miR-30a and miR-106b

\begin{tabular}{lll}
\hline Category & TERM & $P$-value \\
\hline KEGG pathway & Chemokine signaling pathway & $2.60 \mathrm{E}-03$ \\
KEGG pathway & Endocytosis & $6.00 \mathrm{E}-03$ \\
KEGG pathway & Calcium signaling pathway & $2.60 \mathrm{E}-02$ \\
KEGG pathway & Focal adhesion & $3.30 \mathrm{E}-02$ \\
KEGG pathway & p53 signaling pathway & $4.30 \mathrm{E}-02$ \\
\hline
\end{tabular}

model spontaneously develops atherosclerosis that shares features with human hyperlipoproteinemia, and administration of a high-fat diet accelerates atherosclerosis. In the present study, we selected a normal diet to attenuate the eventual influence of the diet on miRNA expression because we had previously confirmed that using 8 weeks of normal diet in apoE $^{-/-}$mice led to characteristic atherosclerosis lesions. ${ }^{15}$

After comparing the plasma miRNA levels of the mice, we observed that some miRNAs were differentially expressed between apoE ${ }^{-1-}$ and control mice. Like in our previous study, ${ }^{15}$ we observed that five miRNAs (mmu-miR-34a, mmumiR-21, mmu-miR-23a, mmu-miR-30a and mmu-miR-106b) were differentially expressed in both plasma and aorta tissue samples. Furthermore, previous studies found differentially expressed miRNAs in different in vitro and in vivo models; ${ }^{17,21,22}$ however, these miRNAs differed from one model to another. In the present study, three of the five miRNAs that were differentially expressed in mice were confirmed in CAD patients. Nevertheless, further studies are necessary to elucidate the connections between these miRNA expression profiles and the mechanism of CAD.
MiRNAs have been shown to be potential markers of a number of cardiovascular diseases, and a recent review summarizes the main results. ${ }^{23}$ These RNAs are considered useful because their levels are relatively stable in serum, and these results have been reproducible. ${ }^{24}$ Particularly in CAD, over 30 different miRNAs have been identified as potential markers. ${ }^{23}$ Some of these miRNAs, for example miR-135a and miR-147, are differentially expressed when compared with healthy controls, ${ }^{25}$ whereas some, such as miR-340 and miR-624 , are differentially expressed in premature CAD. ${ }^{26}$ Some miRNAs, such as miR-133a and miR-499, may assist with predicting elevated risk of developing acute coronary syndrome, ${ }^{27}$ and others, such as miR-135a and miR-147, may help distinguish between patients with stable and unstable angina pectoris. $^{28}$

Of the five miRNAs investigated here, that is, miR-34a, miR-21, miR-23a, miR-30a and miR-106b, to our knowledge, only miR-21 has been shown to have increased levels in acute coronary syndrome related to CAD. ${ }^{29} \mathrm{MiR}-21$ has also been linked to myocardial infarction, with levels either being increased or decreased in affected cardiac tissue, depending on the study. ${ }^{30-32}$ The levels of this miRNA are either increased or decreased in plasma depending on the time point at which samples are taken ${ }^{33,34}$ and are increased in plasma and in biopsies from heart failure patients. ${ }^{35}$ Although we could find no information on a role of miR-30a in CAD, this miRNA has also been linked to myocardial infarction, with increased values in plasma. ${ }^{36}$ Furthermore, increased levels of miR-30a similar to those found in the fetus have been found in serum during heart failure. ${ }^{37}$ Similarly, miR-23a has been associated with late-stage pressure overload-induced hypertrophy and heart failure. ${ }^{38}$ 
CAD is caused by the interaction of a number of genetic and environmental factors. In a previous study, we demonstrated that miRNAs are extracellularly released by exosomes. ${ }^{39}$ Because these miRNAs can be detected in the circulation, they may serve as potential biomarkers of diseases. ${ }^{6}$ We have determined the expression profiles of five miRNAs that are differentially regulated in $\mathrm{apoE}^{-1-}$ mice and in CAD patients (coronary stenosis $>70 \%$ ) and have identified three miRNAs that were differentially regulated in CAD patients. These miRNAs could eventually serve as markers to screen patients or to monitor treatment. However, further studies are needed before these miRNAs will be suitable for clinical use.

We further carried out gene set enrichment analysis to identify the potential targets of these miRNAs. In brief, this process involved the construction of a refined gene set for differentially expressed miRNAs that satisfy the following three criteria: (1) the genes were predicted target genes of the differentially expressed miRNAs; (2) differential expression of the mRNA was identified in previous microarray analysis of apoE $E^{-1-}$ mice; and (3) the differential expression patterns of the mRNAs were negatively correlated to those of the differentially expressed miRNAs. As the results showed, the five miRNAs potentially regulated 117 differentially expressed target genes. The Kyoto Encyclopedia of Genes and Genomes enrichment analysis results are shown in Table 3. Three pathways were enriched with predicted targets of mmumiR-34a, mmu-miR-21, mmu-miR-23a, mmu-miR-30a and mmu-miR-106b that were differently expressed in atherosclerotic tissue compared with control tissue. All three pathways were related to angiogenesis. Indeed, previous studies showed that ectopic expression of miR-34a induced senescence and cell cycle arrest in ECs, and that loss of miR-34 expression was associated with resistance against apoptosis induced by p53-activating agents. ${ }^{40-42}$ MiR-21 was demonstrated to have a role in aberrant vascular smooth muscle cell proliferation, ${ }^{16,43}$ and the inhibition of miR-21 decreased the proliferation and increased the apoptosis of vascular smooth muscle cells. ${ }^{16}$ In support of our results, hsa-miR-21 has been associated with several key processes involved in the progression of atherosclerosis ${ }^{44}$ and in balloon injury in rats. ${ }^{16}$ The miR-2327-24 cluster is enriched in endothelial cells. ${ }^{45}$ As mentioned above, miR-23a is also associated with late-stage pressure overload-induced hypertrophy and heart failure. ${ }^{38}$ The remaining miRNA of the five in this investigation is miR-106b. Although this miRNA, which is within the miR-106b/25 cluster, has so far only been linked to $\mathrm{CAD},{ }^{46}$ it has been shown to be upregulated in the atria of atrial fibrillation patients compared with those with increased sinus rhythm ${ }^{47}$ in hypertension-induced heart failure. ${ }^{48}$ This miRNA is also downregulated during aging ${ }^{49}$ and is a potential marker for various types of cancers. ${ }^{50,51}$ The potential role that this miRNA has in CAD could involve the post-transcriptional regulation of lipid metabolism, ${ }^{52}$ although it has also been suggested that miR-160b is involved in apoptosis and angiogenesis regulation after myocardial infarction. ${ }^{53}$
Although the functions of miR-34a and miR-23a, especially their roles in atherosclerosis, have not been extensively studied, our results and those of previous studies suggest that they may serve as potential indicators of atherosclerosis. Indeed because SIRT1 has been shown to be a direct target of miR-34a, this miRNA could promote aging of endothelial cells by inhibiting SIRT1. MiR-34a also inhibits endothelial progenitor cell (EPC)-mediated angiogenesis by inducing senescence. ${ }^{54,55}$ The number of EPCs, which are involved in new blood vessel formation to maintain endothelial cell homeostasis, is reduced in atherosclerotic patients, indicating that miR-34a may be involved in atherosclerosis progression. ${ }^{54,55}$ In addition, recent evidence suggests that miR-34a and miR-23 may be involved in the p53 pathway, and thus may modulate apoptosis. ${ }^{54-56}$ Indeed, miR-34 has been shown to act with other effectors to inhibit cell proliferation via the p53 pathway. ${ }^{56}$ However, more work is needed on this matter.

Compared with other studies on the role of miRNAs as biomarkers of various diseases, ${ }^{57-59}$ this study contains a relatively small sample size, including only 32 CAD patients and 20 healthy controls. Therefore, these results are more suitable as a pilot study, and we expect that these results will be validated by research on larger populations. However, the identification of three miRNAs that have rarely been described in relation to CAD suggest that further research on these potential markers is warranted.

\section{CONFLICT OF INTEREST}

The authors declare no conflict of interest.

\section{ACKNOWLEDGEMENTS}

This work was supported by grants from the National Natural Science Foundation of China (No: 81270366; No: 81070216). The microarray experiments were performed by Kang Cheng Bio-tech, Shanghai, China.

1 Sun X, Zhang M, Sanagawa A, Mori C, Ito S, Iwaki S et al. Circulating microRNA-126 in patients with coronary artery disease: correlation with LDL cholesterol. Thromb J 2012; 10: 16.

2 Taurino C, Miller WH, McBride MW, McClure JD, Khanin R, Moreno MU et al. Gene expression profiling in whole blood of patients with coronary artery disease. Clin Sci 2010; 119: 335-343.

3 Nissen SE, Tsunoda T, Tuzcu EM, Schoenhagen P, Cooper CJ, Yasin M et al. Effect of recombinant ApoA-I Milano on coronary atherosclerosis in patients with acute coronary syndromes: a randomized controlled trial. JAMA 2003; 290: 2292-2300.

4 Aiello RJ, Brees D, Bourassa PA, Royer L, Lindsey S, Coskran T et al. Increased atherosclerosis in hyperlipidemic mice with inactivation of ABCA1 in macrophages. Arterioscler Thromb Vasc Biol 2002; 22 : 630-637.

5 Joyce CW, Wagner EM, Basso F, Amar MJ, Freeman LA, Shamburek RD et al. ABCA1 overexpression in the liver of LDLr-KO mice leads to accumulation of pro-atherogenic lipoproteins and enhanced atherosclerosis. J Biol Chem 2006; 281: 33053-33065.

6 Chang TC, Mendell JT. microRNAs in vertebrate physiology and human disease. Annu Rev Genomics Hum Genet 2007; 8: 215-239.

7 Lu J, Getz G, Miska EA, Alvarez-Saavedra E, Lamb J, Peck D et al. MicroRNA expression profiles classify human cancers. Nature 2005; 435 : 834-838. 
8 Griffiths-Jones S, Saini HK, van Dongen S, Enright AJ. miRBase: tools for microRNA genomics. Nucleic Acids Res 2008; 36: D154-D158.

9 Wang J, Yang X. The function of miRNA in cardiac hypertrophy. Cell Mol Life Sci 2012; 69: 3561-3570.

10 Bartel DP. MicroRNAs: genomics, biogenesis, mechanism, and function. Cell 2004; 116: 281-297.

11 Marsit CJ, Eddy K, Kelsey KT. MicroRNA responses to cellular stress. Cancer Res 2006; 66: 10843-10848.

12 Brennecke J, Hipfner DR, Stark A, Russell RB, Cohen SM. bantam encodes a developmentally regulated microRNA that controls cell proliferation and regulates the proapoptotic gene hid in Drosophila. Cel/ 2003; 113: 25-36.

13 Artmann S, Jung K, Bleckmann A, Beissbarth T. Detection of simultaneous group effects in microRNA expression and related target gene sets. PLOS ONE 2012; 7: e38365.

14 Fang Y, Shi C, Manduchi E, Civelek M, Davies PF. MicroRNA-10a regulation of proinflammatory phenotype in athero-susceptible endothelium in vivo and in vitro. Proc Natl Acad Sci USA 2010; 107: 13450-13455.

15 Han H, Wang YH, Qu GJ, Sun TT, Li FQ, Jiang W et al. Differentiated miRNA expression and validation of signaling pathways in apoE gene knockout mice by cross-verification microarray platform. Exp Mol Med 2013; 45: e13.

$16 \mathrm{Ji}$ R, Cheng Y, Yue J, Yang J, Liu X, Chen $\mathrm{H}$ et al. MicroRNA expression signature and antisense-mediated depletion reveal an essential role of MicroRNA in vascular neointimal lesion formation. Circ Res 2007; 100: $1579-1588$.

17 Chen T, Huang Z, Wang L, Wang Y, Wu F, Meng S et al. MicroRNA-125a$5 p$ partly regulates the inflammatory response, lipid uptake, and ORP9 expression in oxLDL-stimulated monocyte/macrophages. Cardiovasc Res 2009; 83: 131-139.

18 Chen X, Ba Y, Ma L, Cai X, Yin Y, Wang $\mathrm{K}$ et al. Characterization of microRNAs in serum: a novel class of biomarkers for diagnosis of cancer and other diseases. Cell Res 2008; 18: 997-1006.

19 Gilad S, Meiri E, Yogev Y, Benjamin S, Lebanony D, Yerushalmi N et al. Serum microRNAs are promising novel biomarkers. PLOS ONE 2008; 3: e3148.

20 Bea F, Blessing E, Bennett B, Levitz M, Wallace EP, Rosenfeld ME. Simvastatin promotes atherosclerotic plaque stability in apoE-deficient mice independently of lipid lowering. Arterioscler Thromb Vasc Biol 2002; 22: 1832-1837.

21 Ikeda S, Kong SW, Lu J, Bisping E, Zhang H, Allen PD et al. Altered microRNA expression in human heart disease. Physiol Genomics 2007; 31: 367-373.

22 Raitoharju E, Lyytikainen LP, Levula M, Oksala N, Mennander A, Tarkka M et al. miR-21, miR-210, miR-34a, and miR-146a/b are up-regulated in human atherosclerotic plaques in the Tampere Vascular Study. Atherosclerosis 2011; 219: 211-217.

23 Bronze-da-Rocha E. MicroRNAs expression profiles in cardiovascular diseases. Biomed Res Int 2014; 2014: 985408.

24 Xu J, Zhao J, Evan G, Xiao C, Cheng Y, Xiao J. Circulating microRNAs: novel biomarkers for cardiovascular diseases. J Mol Med 2012; 90: 865-875.

25 Hoekstra M, van der Lans CA, Halvorsen B, Gullestad L, Kuiper J, Aukrust P et al. The peripheral blood mononuclear cell microRNA signature of coronary artery disease. Biochem Biophys Res Commun 2010; 394 792-797.

26 Sondermeijer BM, Bakker A, Halliani A, de Ronde MW, Marquart AA, Tijsen AJ et al. Platelets in patients with premature coronary artery disease exhibit upregulation of miRNA340* and miRNA624*. PloS one 2011; 6: e25946.

27 De Rosa S, Fichtlscherer S, Lehmann R, Assmus B, Dimmeler S, Zeiher AM. Transcoronary concentration gradients of circulating microRNAs. Circulation 2011: 124: 1936-1944.

28 D'Alessandra Y, Carena MC, Spazzafumo L, Martinelli F, Bassetti B, Devanna $\mathrm{P}$ et al. Diagnostic potential of plasmatic MicroRNA signatures in stable and unstable angina. PloS one 2013; 8: e80345.

29 Diehl P, Fricke A, Sander L, Stamm J, Bassler N, Htun N et al. Microparticles: major transport vehicles for distinct microRNAs in circulation. Cardiovasc Res 2012; 93: 633-644.

30 Thum T, Galuppo P. Wolf C, Fiedler J, Kneitz S, van Laake LW et al. MicroRNAs in the human heart: a clue to fetal gene reprogramming in heart failure. Circulation 2007; 116: 258-267.

31 Dong S, Cheng Y, Yang J, Li J, Liu X, Wang X et al. MicroRNA expression signature and the role of microRNA-21 in the early phase of acute myocardial infarction. J Biol Chem 2009; 284: 29514-29525.

32 Cheng Y, Zhu P, Yang J, Liu X, Dong S, Wang X et al. Ischaemic preconditioning-regulated miR-21 protects heart against ischaemia/ reperfusion injury via anti-apoptosis through its target PDCD4. Cardiovasc Res 2010; 87: 431-439.

33 Zile MR, Mehurg SM, Arroyo JE, Stroud RE, DeSantis SM, Spinale FG. Relationship between the temporal profile of plasma microRNA and left ventricular remodeling in patients after myocardial infarction. Circ Cardiovasc Genet 2011; 4: 614-619.

34 Olivieri F, Antonicelli R, Capogrossi MC, Procopio AD. Circulating microRNAs (miRs) for diagnosing acute myocardial infarction: an exciting challenge. Int J Cardiol 2013; 167: 3028-3029.

35 Villar AV, García R, Merino D, Llano M, Cobo M, Montalvo C et al. Myocardial and circulating levels of microRNA-21 reflect left ventricular fibrosis in aortic stenosis patients. Int J Cardiol 2013; 167: 2875-2881.

36 Long G, Wang F, Duan Q, Yang S, Chen F, Gong W et al. Circulating miR-30a, miR-195 and let-7b associated with acute myocardial infarction. PloS one 2012; 7: e50926.

37 Zhao DS, Chen Y, Jiang H, Lu JP, Zhang G, Geng J et al. Serum miR-210 and miR-30a expressions tend to revert to fetal levels in Chinese adult patients with chronic heart failure. Cardiovasc Pathol 2013; 22: 444-450.

38 Busk PK, Cirera S. MicroRNA profiling in early hypertrophic growth of the left ventricle in rats. Biochem Biophys Res Commun 2010; 396: 989-993.

39 Luo SS, Ishibashi O, Ishikawa G, Ishikawa T, Katayama A, Mishima T et al. Human villous trophoblasts express and secrete placenta-specific microRNAs into maternal circulation via exosomes. Biol Reprod 2009; 81: 717-729.

40 Fujita Y, Kojima K, Hamada N, Ohhashi R, Akao Y, Nozawa $Y$ et al. Effects of miR-34a on cell growth and chemoresistance in prostate cancer PC3 cells. Biochem Biophys Res Commun 2008; 377: 114-119.

41 Lodygin D, Tarasov V, Epanchintsev A, Berking C, Knyazeva T, Korner H et al. Inactivation of miR-34a by aberrant $\mathrm{CpG}$ methylation in multiple types of cancer. Cell cycle 2008; 7: 2591-2600.

42 Hermeking H. The miR-34 family in cancer and apoptosis. Cell Death Differ 2010; 17: 193-199.

43 Liu X, Cheng Y, Zhang S, Lin Y, Yang J, Zhang C. A necessary role of miR-221 and miR-222 in vascular smooth muscle cell proliferation and neointimal hyperplasia. Circ Res 2009; 104: 476-487.

44 Weber M, Baker MB, Moore JP, Searles CD. MiR-21 is induced in endothelial cells by shear stress and modulates apoptosis and eNOS activity. Biochem Biophys Res Commun 2010; 393: 643-648.

45 Zhou Q, Gallagher R, Ufret-Vincenty R, Li X, Olson EN, Wang S. Regulation of angiogenesis and choroidal neovascularization by members of microRNA-23 27 24 clusters. Proc Natl Acad Sci USA 2011; 108: 8287-8292.

46 Ren J, Zhang J, Xu N, Han G, Geng Q, Song J et al. Signature of circulating microRNAs as potential biomarkers in vulnerable coronary artery disease. PloS one 2013; 8: e80738.

47 Slagsvold KH, Johnsen AB, Rognmo O, Høydal M, Wisløff U, Wahba A Comparison of left versus right atrial myocardium in patients with sinus rhythm or atrial fibrillation - an assessment of mitochondrial function and microRNA expression. Physiol Rep 2014; 2: e12124.

48 Dickinson BA, Semus HM, Montgomery RL, Stack C, Latimer PA, Lewton $\mathrm{SM}$ et al. Plasma microRNAs serve as biomarkers of therapeutic efficacy and disease progression in hypertension-induced heart failure. Eur $\mathrm{J}$ Heart Fail 2013; 15: 650-659.

49 Zhang $\mathrm{H}$, Yang $\mathrm{H}$, Zhang $\mathrm{C}$, Jing $\mathrm{Y}$, Wang $\mathrm{C}$, Liu $\mathrm{C}$ et al. Investigation of MicroRNA Expression in Human Serum During the Aging Process. J Gerontol A Biol Sci Med Sci 2014, pii: glu145.

$50 \mathrm{Ni}$ X, Xia T, Zhao Y, Zhou W, Wu N, Liu X et al. Downregulation of miR-106b induced breast cancer cell invasion and motility in association with overexpression of matrix metalloproteinase 2. Cancer Sci 2014; 105: 18-25.

51 Wang JL, Hu Y, Kong $\mathrm{X}$, Wang $\mathrm{ZH}$, Chen $\mathrm{HY}, \mathrm{Xu} \mathrm{J}$ et al. Candidate microRNA biomarkers in human gastric cancer: a systematic review and validation study. PloS one 2013; 8: e73683.

52 Rotllan N, Fernández-Hernando C. MicroRNA Regulation of Cholesterol Metabolism. Cholesterol 2012; 2012: 847849.

53 Liu Z, Yang D, Xie P, Ren G, Sun G, Zeng X et al. MiR-106b and MiR-15b modulate apoptosis and angiogenesis in myocardial infarction. Cell Physiol Biochem 2012; 29: 851-862.

54 Luan S, Sun L, Huang F. MicroRNA-34a: a novel tumor suppressor in p53mutant glioma cell line U251. Arch Med Res 2010; 41: 67-74.

55 Aranha MM, Santos DM, Sola S, Steer CJ, Rodrigues CM. miR-34a regulates mouse neural stem cell differentiation. PLOS ONE 2011; 6 : e21396. 
56 He L, He X, Lim LP, de Stanchina E, Xuan Z, Liang Y et al. A microRNA component of the p53 tumour suppressor network. Nature 2007; 447: 1130-1134.

57 Schrauder MG, Strick R, Schulz-Wendtland R, Strissel PL, Kahmann L, Loehberg CR et al. Circulating micro-RNAs as potential blood-based markers for early stage breast cancer detection. PloS one 2012; 7: e29770.

$58 \mathrm{Ng}$ EK, Li R, Shin VY, Jin HC, Leung CP, Ma ES et al. Circulating microRNAs as specific biomarkers for breast cancer detection. PloS one 2013; 8: e53141.

59 Hennessey PT, Sanford T, Choudhary A, Mydlarz WW, Brown D, Adai AT et al. Serum microRNA biomarkers for detection of non-small cell lung cancer. PloS one 2012; 7: e32307. (c) (i) (2) (2) This work is licensed under a Creative Commons Attribution-NonCommercial-ShareAlike

3.0

Unported License. The images or other third party material in this article are included in the article's Creative Commons license, unless indicated otherwise in the credit line; if the material is not included under the Creative Commons license, users will need to obtain permission from the license holder to reproduce the material. To view a copy of this license, visit http://creativecommons.org/licenses/by-nc-sa/3.0/ 